Robert D. Guber, BS*

Varun Takyar, MD*

Angela Kokkinis, BSN, RN

Derrick A. Fox, MD

Hawwa Alao, MD

Ilona Kats, BA

Dara Bakar, BA

Alan T. Remaley, MD,

$\mathrm{PhD}$

Stephen M. Hewitt, MD, $\mathrm{PhD}$

David E. Kleiner, MD, $\mathrm{PhD}$

Chia-Ying Liu, PhD

Colleen Hadigan, MD

Kenneth H. Fischbeck, MD

Yaron Rotman, MD

Christopher Grunseich, MD

Correspondence to

Dr. Grunseich:

christopher.grunseich@nih.gov

Supplemental data at Neurology.org

\title{
Nonalcoholic fatty liver disease in spinal and bulbar muscular atrophy OPEN
}

ABSTRACT

Objective: To determine the prevalence and features of fatty liver disease in spinal and bulbar muscular atrophy (SBMA).

Methods: Two groups of participants with SBMA were evaluated. In the first group, 22 participants with SBMA underwent laboratory analysis and liver imaging. In the second group, 14 participants with SBMA were compared to 13 female carriers and 23 controls. Liver biopsies were done in 4 participants with SBMA.

Results: Evidence of fatty liver disease was detected by magnetic resonance spectroscopy in all participants with SBMA in the first group, with an average dome intrahepatic triacylglycerol of 27\% (range 6\%-66\%, ref $\leq 5.5 \%$ ). Liver dome magnetic resonance spectroscopy measurements were significantly increased in participants with SBMA in the second group relative to age- and sex-matched controls, with average disease and male control measurements of $17 \%$ and $3 \%$, respectively. Liver biopsies were consistent with simple steatosis in 2 participants and nonalcoholic steatohepatitis in 2 others.

Conclusions: We observed evidence of nonalcoholic liver disease in nearly all of the participants with SBMA evaluated. These observations expand the phenotypic spectrum of the disease and provide a potential biomarker that can be monitored in future studies. Neurology ${ }^{\circledR}$ 2017;89:2481-2490

\section{GLOSSARY}

$\mathbf{A L T}=$ alanine aminotransferase; $\mathbf{A R}=$ androgen receptor; $\mathbf{B M I}=$ body mass index; $\mathbf{C K}=$ creatine kinase; $\mathbf{d d P C R}=$ digital droplet PCR; HOMA-IR = Homeostatic Model for Assessment of Insulin Resistance; IGF-1 = insulin-like growth factor 1; IHTG = intrahepatic triacylglycerol; MRS = magnetic resonance spectroscopy; NAFLD = nonalcoholic fatty liver disease; $\mathbf{N A S H}=$ nonalcoholic steatohepatitis; NFS = NAFLD Fibrosis Score; RBP4 = retinol binding protein 4; SBMA = spinal and bulbar muscular atrophy; TG = triacylglycerol.

Spinal and bulbar muscular atrophy (SBMA) is a hereditary adult-onset neuromuscular disease primarily affecting men. ${ }^{1}$ It is caused by a CAG repeat expansion mutation in the androgen receptor (AR) gene on the $\mathrm{X}$ chromosome. ${ }^{2}$ Patients with SBMA develop slowly progressive extremity and facial weakness, typically in the 4 th or 5 th decade of life. ${ }^{3,4}$ Female carriers of the disease gene are generally not symptomatic. ${ }^{5}$

SBMA likely involves both a loss of normal function and a toxic gain of function of the mutant AR. The gain of function is characterized by toxicity to muscle and nerve, and is dependent on AR ligand (dihydrotestosterone or testosterone) in cell and animal models. ${ }^{6}$ Although the neuromuscular findings and androgen insensitivity of SBMA are well-known, there are other non-neural features of the disease that have only been described recently. Abnormal bladder function and bone mineral density have been reported in patients with SBMA. ${ }^{7}$ ECG findings of Brugada syndrome were detected in $49 \%$ of patients from a large Japanese cohort. ${ }^{8}$ Features of

\footnotetext{
*These authors contributed equally to this work.

From the Neurogenetics Branch (R.D.G., A.K., D.A.F., I.K., D.B., K.H.F., C.G.), National Institute of Neurological Disorders and Stroke; Liver Diseases Branch (V.T., H.A., Y.R.), National Institute of Diabetes and Digestive and Kidney Diseases; Cardiovascular and Pulmonary Branch (A.T.R.), National Heart Lung \& Blood Institute; Laboratory of Pathology (S.M.H., D.E.K.), National Cancer Institute; Radiology and Imaging Sciences (C.-Y.L.), Clinical Center, National Institute of Allergy and Infectious Diseases; and NIH (C.H.), Bethesda, MD.

Go to Neurology.org for full disclosures. Funding information and disclosures deemed relevant by the authors, if any, are provided at the end of the article. The Article Processing Charge was funded by the National Institute of Neurological Disorders and Stroke.

This is an open access article distributed under the terms of the Creative Commons Attribution-NonCommercial-NoDerivatives License 4.0 (CC BY-NC-ND), which permits downloading and sharing the work provided it is properly cited. The work cannot be changed in any way or used commercially without permission from the journal.
} 
metabolic dysfunction with elevated serum levels of triglycerides, cholesterol, and glucose have been reported. ${ }^{4,7}$ In addition, measures of insulin resistance were found to be increased in a cohort of 55 patients with SBMA. ${ }^{?}$

Nonalcoholic fatty liver disease (NAFLD), the accumulation of excess fat in the liver, is currently the most common liver disorder in the Western world and is tightly associated with obesity and the metabolic syndrome. ${ }^{10}$ Although in many cases NAFLD is limited to fat deposition (steatosis), in a subset of patients the fat accumulation is associated with liver injury and inflammation (nonalcoholic steatohepatitis [NASH]), with resultant liver fibrosis and potential to progress to cirrhosis. ${ }^{11}$ NASH can only be differentiated from steatosis on the basis of histologic evaluation, requiring a liver biopsy for full evaluation. ${ }^{12-14}$

We have noted liver imaging consistent with fatty liver in participants with SBMA seen at our institution. The aim of this study was to formally investigate our hypothesis that NAFLD is a manifestation of SBMA.

METHODS Standard protocol approvals, registrations, and patient consents. Participants were recruited under $\mathrm{NIH}$ protocols NCT01369901 and NCT00004568 (group A) and NCT02124057 (group B). The study was approved by the NIH Combined Neuroscience Institutional Review Board, and informed written consent was obtained from all participants.

Patients and clinical evaluation. Two groups of participants with SBMA were evaluated at the NIH Clinical Center. Group A included 22 participants with SBMA seen for clinical evaluation and an exercise trial from 2013-2014. Because the high prevalence of NAFLD in the general population necessitated comparison to controls, we subsequently initiated and enrolled participants in another observational study (group B). The study enrolled 14 patients with SBMA, 13 female carriers, and 23 controls from 2014 to 2017 . The data collected from group A were used to calculate a sample size needed to test for a difference in liver fat between participants with SBMA and male controls with a power of $80 \%$ and significance level of 0.05 . Of the 23 controls, 14 were male controls who were agematched ( \pm 5 years) to the participants with SBMA and the other 9 were female controls age-matched to the SBMA carriers. All were screened by a neurologist and hepatologist to evaluate their eligibility for the study. Participants with SBMA and carriers were genetically diagnosed before enrollment, and CAG repeat length measurements were obtained from a Clinical Laboratory Improvement Amendments-certified laboratory. Participants with SBMA and controls were excluded from participation if they had a history of nonfatty liver disease, heavy alcohol consumption (greater than 1 drink per day for women and 2 drinks per day for men), ${ }^{15}$ or an acquired condition that would be expected to affect liver function, such as viral or autoimmune hepatitis.
Laboratory analysis. Fasting blood was collected and analyzed by the NIH Clinical Center Department of Laboratory of Medicine for standard liver and metabolic tests as well as screening tests for viral hepatitis and other liver diseases. Serum samples from participants in group A were centrifuged for 5 minutes at $2,000 \times g$, stored at $-80^{\circ} \mathrm{C}$, and then batch tested for insulinlike growth factor-binding protein-3 using the IMMULITE IGFBP-3 assay test (Siemens Medical Solutions; Malvern, PA).

Liver imaging. Liver and muscle fat content was assessed by ${ }^{1} \mathrm{H}$ magnetic resonance spectroscopy (MRS). ${ }^{16}$ Triacylglycerol (TG) content was quantified at 3 standard voxel localizations $(3 \times 3 \times$ $3 \mathrm{~cm}$ ), including the dome of the right hepatic lobe, the right inferior lobe, and the left lobe. The measurement of the dome of the right hepatic lobe was primarily used for measurement of fat content, as this location has been shown to have optimal intrahepatic voxel placement and to yield high-quality spectra. ${ }^{16}$ Proton density fat fraction was calculated from the integral peak areas with a standardized formula. ${ }^{17}$ TG content of the thigh muscles was similarly calculated as an average of the quadriceps and hamstring muscle voxel locations. MRS data were analyzed unblinded in group A and blinded in group B. In addition, semiquantitative estimation of steatosis was performed in a blinded manner using ultrasound by a trained radiologist. Participants who had evidence of steatosis on either imaging modality were defined as having fatty liver.

Liver samples. Participants with SBMA with persistent elevation of transaminases and imaging findings of fatty liver disease were offered a liver biopsy. Biopsy samples were divided with one aliquot stored in RNALater for RNA analysis, one aliquot formalin-fixed for standard histologic evaluation, and a third aliquot flash-frozen in liquid nitrogen. For gene expression analysis, we compared the participants with SBMA to age- and sex-matched NASH and control liver samples from the Liver Tissue Cell Distribution System (Minneapolis, MN), which is funded by NIH Contract HHSN276201200017C. For immunohistochemical assays, the SBMA samples were compared to 2 control adult male biopsy samples.

Hepatic gene expression. Total RNA was extracted from pulverized frozen samples using TRIzol Reagent per manufacturer recommendations (ThermoFisher Scientific, Waltham, MA). All the samples had a 280/260 ratio $\geq 1$.9. cDNA was generated from $250 \mathrm{ng}$ of purified RNA using the RT ${ }^{2}$ First Strand Kit (Qiagen, Venlo, the Netherlands) protocol. The Fatty Liver RT ${ }^{2}$ Profiler PCR Array (PAHS-157 ZE, Qiagen) was used to evaluate fatty liver signaling pathways using 3 control liver samples, 3 NASH liver samples, and 3 of the SBMA liver biopsies we obtained. The $\mathrm{Ct}$ value was normalized to the average of 5 housekeeping genes in the array. Fold change was calculated based on the average of the normalized Ct values. Pathways were selected according to the manufacturer's recommendations; a given gene may be included in more than one pathway.

Hepatic expression of the AR was quantified in $2 \mathrm{ng}$ of cDNA using digital droplet PCR (ddPCR) on the Bio-Rad QX200 Droplet Digital PCR System (Bio-Rad, Hercules, CA) and a commercially available probe (dHsaCPE5047114; Bio-Rad). The reaction was run using the manufacturer's settings. The results were analyzed using QuantaSoft Software (Bio-Rad).

Histology and AR staining of liver biopsies. Formalin-fixed liver tissue was sectioned and stained with hematoxylin \& eosin, Masson trichrome, and both ubiquitin (Dako, Santa Cruz, CA) and AR (Santa Cruz Biotechnology, Dallas, TX) antibodies. Histology slides were scored for histologic features of fatty liver 
disease by an experienced hepatopathologist (D.E.K.) according to the NASH Clinical Research Network scoring system. ${ }^{18}$

Statistical analysis. Comparisons between disease and control populations were performed using a 2-tailed Student $t$ test with $p<0.05$ to determine significance. Univariate correlation analysis was performed using a linear regression model to calculate the $R^{2}$ goodness of fit and corresponding $p$ value. Multivariate linear regression was performed using SPSS Statistics v.19 (IBM, Armonk, NY).

RESULTS Prevalence of fatty liver in SBMA. Twentytwo male participants with SBMA completed clinical evaluation, including liver imaging and laboratory testing in group A (table 1). The average age was 54 years and all had symptomatic weakness at the time of evaluation. Alanine aminotransferase (ALT) was elevated $(>30 \mathrm{U} / \mathrm{L})$ in $21(96 \%)$ and creatine kinase (CK) was elevated in 20 (91\%). Overall, fatty liver (hepatic steatosis) was found in all 22 participants by at least one imaging modality. Twelve of the 16

\begin{tabular}{|c|c|c|c|}
\hline \multirow[t]{2}{*}{ Table 1} & \multicolumn{3}{|c|}{$\begin{array}{l}\text { Group A spinal and bulbar muscular atrophy (SBMA) patient } \\
\text { characteristics }\end{array}$} \\
\hline & & SBMA $(n=22)$ & Reference range \\
\hline Age, y & & $54 \pm 12$ & - \\
\hline \multicolumn{2}{|c|}{ CAG repeat number } & $48 \pm 5$ & $<38$ \\
\hline \multicolumn{2}{|c|}{$\mathrm{BMI}, \mathbf{k g} / \mathrm{m}^{2}$} & $26 \pm 4$ & - \\
\hline \multicolumn{2}{|c|}{ Platelet count, $\mathrm{K} / \mu \mathrm{L}$} & $236 \pm 44$ & $161-347$ \\
\hline \multicolumn{2}{|c|}{ Albumin, $\mathrm{g} / \mathrm{dL}$} & $4.1 \pm 0.3$ & $3.5-5.2$ \\
\hline \multicolumn{2}{|c|}{ Total bilirubin, $\mathrm{mg} / \mathrm{dL}$} & $0.5 \pm 0.2$ & $0.0-1.2$ \\
\hline \multicolumn{2}{|l|}{ CK, U/L } & $1174 \pm 840[73-3471]$ & 39-308 \\
\hline \multicolumn{2}{|l|}{ ALT, U/L } & $70 \pm 27[26-127]$ & $0-41$ \\
\hline \multicolumn{2}{|l|}{ AST, U/L } & $51 \pm 24$ [15-107] & $0-40$ \\
\hline \multicolumn{2}{|c|}{ IGF-1, ng/mL } & $149 \pm 50$ & $101-267$ \\
\hline \multicolumn{2}{|c|}{ IGFBP3, ng/mL } & $4.7 \pm 1.2$ & $3.0-6.9$ \\
\hline \multicolumn{2}{|c|}{ Glucose, mg/dL } & $109 \pm 30$ & $74-106$ \\
\hline \multirow{3}{*}{\multicolumn{2}{|c|}{ Liver ultrasound }} & 19\% Mild (3) & Normal \\
\hline & & $38 \%$ Moderate (6) & \\
\hline & & 19\% Severe (3) & \\
\hline \multicolumn{2}{|c|}{ IHTG \% (dome) } & $27 \pm 17[6-66], n=18$ & $<5$ \\
\hline \multicolumn{2}{|c|}{ IHTG \% (inferior lobe) } & $25 \pm 16$ [6-62], $n=19$ & $<5$ \\
\hline \multicolumn{2}{|c|}{ IHTG \% (left lobe) } & $25 \pm 15[6-59], n=17$ & $<5$ \\
\hline
\end{tabular}

Abbreviation: $\mathrm{BMI}=$ body mass index.

Data shown are the average \pm SD for the cohort of 22 participants unless otherwise specified within the data column. Platelet count, albumin, glucose, total bilirubin, aspartate aminotransferase (AST), alanine aminotransferase (ALT), creatine kinase (CK), and insulinlike growth factor 1 (IGF-1) reference ranges from the NIH Department of Laboratory Medicine. Insulin-like growth factor binding protein 3 (IGFBP3) reference ranges from Mayo Clinical Labs (Rochester, MN). Ultrasound findings graded as mild, moderate, or severe with the number of participants in each category indicated in parentheses. Magnetic resonance liver spectroscopy intrahepatic triacylglycerol content (IHTG) measurements calculated from the dome, inferior, and left lobes. This cohort comprised 16 Caucasian, 2 Asian, 1 African American, 1 Hispanic, 1 American Indian, and 1 multiple-race participants. Ranges for IHTG, CK, AST, and ALT indicated with brackets. Laboratory and imaging assessments performed at the NIH Clinical Center. participants who had liver ultrasound were found to have steatosis, with 3 (19\%) assessed as mild, 6 (38\%) moderate, and 3 (19\%) severe. MRS performed in 19 participants showed liver dome intrahepatic triacylglycerol (IHTG) $>5.5 \%$ in all who were evaluated (range 6\%-66\%).

To further investigate the finding of fatty liver disease in SBMA, we conducted another observational study in a separate group of 14 participants with SBMA (group B) together with SBMA carriers and age- and sex-matched controls (table 2). The SBMA, carrier, and female control groups were composed of greater than $80 \%$ Caucasian participants, with the male control group having approximately $50 \%$ African American and 50\% Caucasian participants. Similar to group A, ultrasound showed steatosis in 11 of the 14 participants with SBMA (79\%), while MRS showed liver dome IHTG $>5.5 \%$ in 11 (79\%; figure 1 , A and B). All but one participant with SBMA in group $\mathrm{B}$ was found to have steatosis by at least one imaging modality. ALT was elevated in 10 (71\%) and CK in 12 (86\%) participants.

Clinical features in participants with SBMA. To evaluate clinical features in participants with SBMA in relation to hepatic steatosis, we combined patients from the 2 groups $(\mathrm{n}=36)$. ALT correlated with CK $(r=0.63$, $p<0.0001$; figure 1C), but showed no association with liver dome IHTG $(r=0.31, p=0.09$; figure 1D). Similarly, on multivariate regression with both CK and IHTG, ALT was significantly associated with CK $(p<0.001)$ but not with liver dome IHTG $(p=0.29)$.

Liver dome IHTG was significantly associated with body mass index (BMI) $(r=0.44, p=0.01$; figure $1 \mathrm{E}$ ), as expected. Interestingly, liver dome IHTG was also $>5.5 \%$ in 8 of the 9 participants with normal BMI $\left(<25 \mathrm{~kg} / \mathrm{m}^{2}\right)$. On univariate analysis, liver dome IHTG showed no significant association with the length of the CAG repeat in the AR gene $(r=0.32, p=0.08$; figure $1 \mathrm{~F})$. On multivariate analysis with BMI and CAG repeat length, liver dome IHTG was independently associated with both BMI $(p=0.009)$ and CAG repeat length $(p=0.06)$. Thus, the known association of liver fat content with BMI may be modulated in SBMA by the severity of the genetic abnormality. The NAFLD Fibrosis Score (NFS) was suggested as a noninvasive tool to identify substantial liver fibrosis in patients with NAFLD ${ }^{19}$ In the SBMA groups, only 1 (3\%) participant had NFS $>0.676$, suggesting advanced fibrosis; 24 participants (69\%) had NFS $<-1.455$, suggesting low risk of advanced fibrosis; and 10 (29\%) had intermediate values.

We compared the participants with SBMA in group $\mathrm{B}$ to age- and sex-matched controls. As 
Table 2 Group B liver study characteristics

\begin{tabular}{|c|c|c|c|c|}
\hline & SBMA (male), $n=14$ & Carrier (female), $n=13$ & Control (male), $n=14$ & Control (female), $n=9$ \\
\hline Age, y & $58 \pm 8$ & $45 \pm 17$ & $59 \pm 8$ & $42 \pm 15$ \\
\hline CAG repeat number & $45 \pm 2$ & $47 \pm 4$ & - & - \\
\hline $\mathrm{BMI}, \mathrm{kg} / \mathrm{m}^{2}$ & $27 \pm 2$ & $25 \pm 4$ & $26 \pm 3$ & $24 \pm 2$ \\
\hline Ethnicity & $93 \% \mathrm{C}, 7 \% \mathrm{PI}$ & $92 \%$ C, 8\% M & $50 \%$ AA, $43 \%$ C, $7 \%$ A & $78 \% \mathrm{C}, 11 \% \mathrm{H}, 11 \% \mathrm{AA}$ \\
\hline Platelet count, $\mathrm{K} / \mu \mathrm{L}$ & $239 \pm 62$ & $256 \pm 52$ & $214 \pm 38$ & $241 \pm 36$ \\
\hline Albumin, g/dL & $4.3 \pm 0.2$ & $4.4 \pm 0.3$ & $4.4 \pm 0.2$ & $4.3 \pm 0.4$ \\
\hline Total bilirubin, mg/dL & $0.4 \pm 0.2$ & $0.4 \pm 0.3$ & $0.7 \pm 0.3$ & $0.4 \pm 0.2$ \\
\hline CK, U/L & $860 \pm 439[240-1500]^{a}$ & $119 \pm 84$ [46-372] & $205 \pm 122$ [71-420] & $200 \pm 317[59-1039]^{\mathrm{b}}$ \\
\hline ALT, U/L & $40 \pm 17[20-80]^{a}$ & $31 \pm 29$ [9-93] & $21 \pm 6[12-28]$ & $16 \pm 7[10-28]$ \\
\hline AST, U/L & $35 \pm 12[17-59]^{c}$ & $31 \pm 29[13-114]$ & $23 \pm 7$ [14-39] & $21 \pm 10[13-45]$ \\
\hline GGT, U/L & $22 \pm 9$ & $19 \pm 7$ & $30 \pm 13$ & $18 \pm 12$ \\
\hline IGF-1, ng/mL & $136 \pm 60$ & $182 \pm 61$ & $163 \pm 63$ & $202 \pm 87$ \\
\hline Cholesterol, mg/dL & $204 \pm 43$ & $188 \pm 27$ & $193 \pm 38$ & $192 \pm 31$ \\
\hline Triglycerides, mg/dL ${ }^{d}$ & $145 \pm 77^{e}$ & $105 \pm 32$ & $86 \pm 38$ & $87 \pm 34$ \\
\hline Free fatty acids, $\mathrm{mEq} / \mathrm{L}$ & $0.44 \pm 0.15$ & $0.33 \pm 0.23$ & $0.43 \pm 0.26$ & $0.38 \pm 0.12$ \\
\hline Insulin, $\mu \mathrm{IU} / \mathrm{mL}$ & $22 \pm 15^{e}$ & $11 \pm 9$ & $10 \pm 9$ & $9 \pm 4$ \\
\hline Glucose, mg/dL & $115 \pm 14^{a}$ & $99 \pm 14$ & $97 \pm 10$ & $93 \pm 9$ \\
\hline HOMA-IR & $6.4 \pm 4.8^{c}$ & $2.9 \pm 2.9$ & $2.3 \pm 1.7$ & $2.0 \pm 0.9$ \\
\hline \multirow[t]{3}{*}{ Liver ultrasound } & $36 \%$ Mild (5) & $31 \%$ Mild (4) & 14\% Mild (2) & $100 \%$ Normal \\
\hline & 29\% Moderate (4) & & & \\
\hline & 14\% Severe (2) & $8 \%$ Severe (1) & & \\
\hline IHTG \% (dome) & $17 \pm 14[3-44]^{\mathrm{c}}$ & $4 \pm 7[0.5-24]$ & $3 \pm 3[0.5-10]$ & $2 \pm 2[0.5-6]$ \\
\hline IHTG \% (inferior lobe) & $15 \pm 13[3-44]^{c}$ & $3 \pm 7[0.5-27]$ & $3 \pm 3[0.5-9]$ & $1 \pm 1[0.5-4]$ \\
\hline IHTG \% (left lobe) & $13 \pm 12[2-36]^{\mathrm{c}}$ & $1 \pm 2[0.5-7]$ & $4 \pm 3[0.5-12]$ & $2 \pm 2[0.5-8]$ \\
\hline Intramuscular triglyceride content \% (thigh) & $30 \pm 24^{a}$ & $6 \pm 3$ & $6 \pm 4$ & $4 \pm 2$ \\
\hline
\end{tabular}

Table 2 clinical characteristics of the liver study group B. Data shown are the average \pm SD for spinal and bulbar muscular atrophy (SBMA) group B, SBMA carriers, and male and female controls. Androgen receptor CAG repeat number, body mass index (BMI), total bilirubin, alanine aminotransferase (ALT), aspartate aminotransferase (AST), creatine kinase (CK), $\gamma$-glutamyl transferase (GGT), insulin-like growth factor 1 (IGF-1), growth hormone (GH), and homeostatic model assessment insulin resistance index (HOMA-IR). Ultrasound findings graded as mild, moderate, or severe with the number of participants in each category indicated in parentheses. Percentage of ethnicity present within each cohort as indicated by Pacific Islander (PI), Caucasian (C), mixed (M), Asian (A), Hispanic (H), and African American (AA). Magnetic resonance liver spectroscopy intrahepatic triacylglycerol content (IHTG) calculated from the dome, inferior, and left lobes. Ranges for IHTG, CK, AST, and ALT indicated with brackets.

${ }^{a} p \leq 0.001$.

${ }^{b}$ Female control with $\mathrm{CK}$ of $1,039 \mathrm{U} / \mathrm{L}$ had a period of increased physical activity before laboratory testing.

${ }^{\mathrm{c}} \mathrm{p} \leq 0.01$.

${ }^{\mathrm{d}}$ Analysis shown excludes one patient with SBMA with hypertriglyceridemia.

${ }^{e} p \leq 0.05$.

expected, those with SBMA had significantly higher serum levels of CK, ALT, and aspartate aminotransferase $(p<0.01)$ (table 2). Hepatic steatosis by MRS (IHTG > 5.5\%) was seen in 11 of 14 (79\%) participants with SBMA compared to 2 of 14 controls $(14 \%, p=0.002)$, despite similar BMIs. The SBMA cohort also had evidence of insulin resistance and metabolic syndrome with higher fasting glucose and insulin compared to controls. Consistent with this finding, the Homeostatic Model for Assessment of Insulin Resistance (HOMA-IR) was significantly higher in SBMA.
Similarly, serum triglycerides were significantly elevated in patients with SBMA compared to controls. Female carriers had muscle and liver enzymes that were not different from those of controls and no evidence of insulin resistance. Although 39\% of carriers had abnormal findings on ultrasound, only low rates of steatosis were detected by MRS (2 of 13 [15\%]; figure $1 \mathrm{~A}$ ).

Muscle fat deposition. SBMA is known to result in muscle degeneration, and thigh muscle MRS demonstrated fatty infiltration (table 2). Muscle fat deposition 
Figure 1 Increased steatosis in spinal and bulbar muscular atrophy (SBMA)
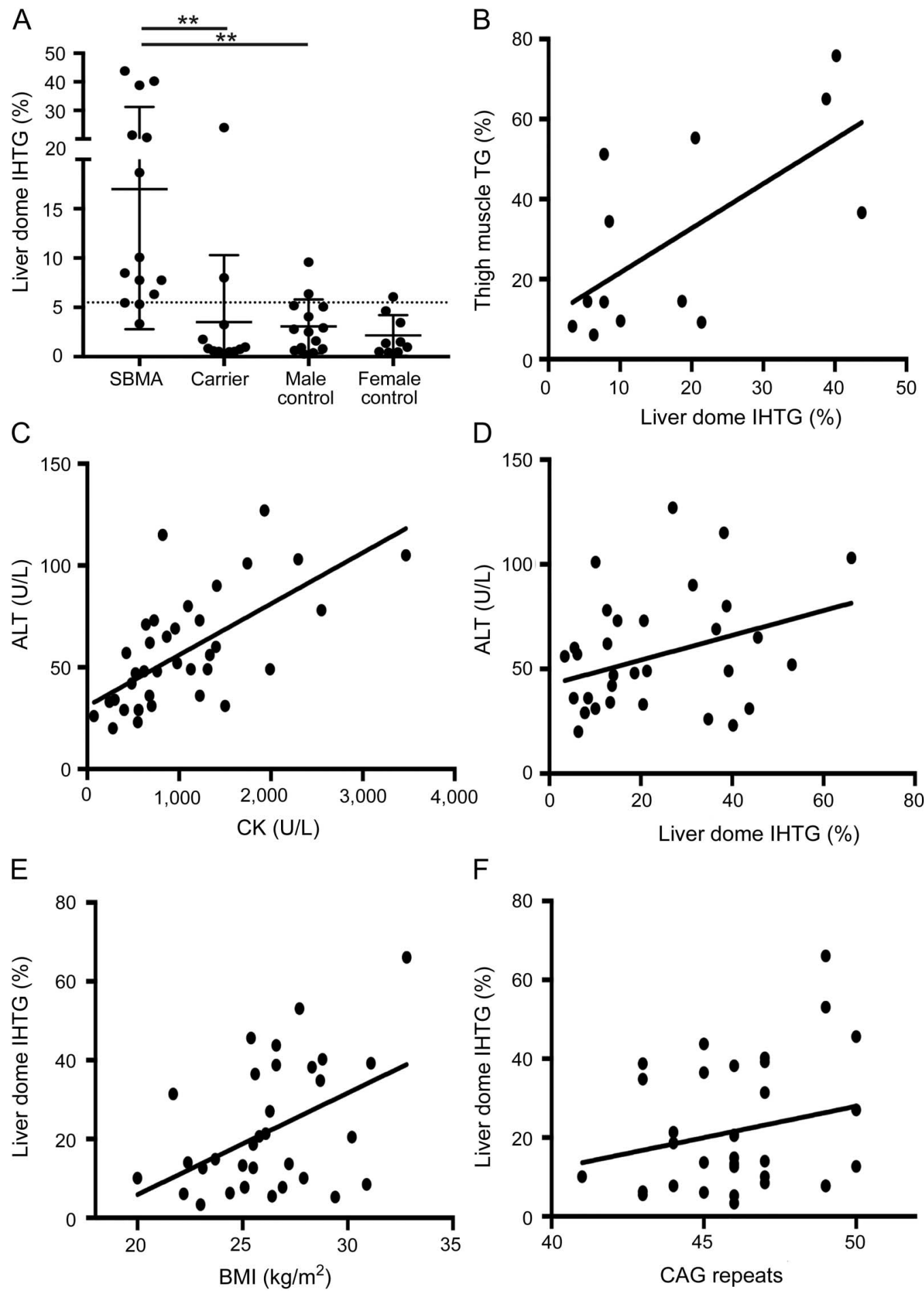

$\mathrm{F}$

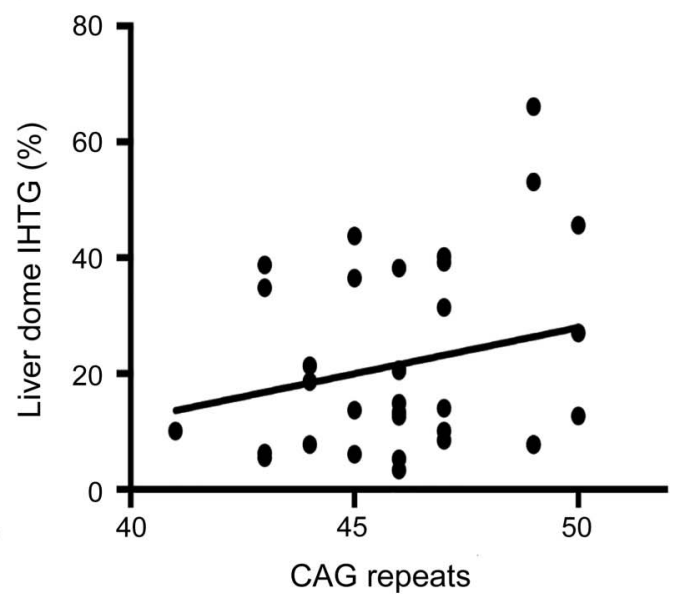

${ }^{1} \mathrm{H}$ magnetic resonance spectroscopic imaging shows intrahepatic triacylglycerol (IHTG) content in the dome of the liver in the SBMA, SBMA carrier, and control groups. The SBMA group has significantly more liver dome IHTG than either the carrier or control groups $(\mathrm{A})$; $* * \mathrm{p}<0.01$; dotted line indicates $5.5 \%$ reference control value. There is a significant correlation between the intramuscular triacylglycerol (TG) content and liver dome IHTG within the SBMA group ( $r=0.43, p=0.01)(B)$. Correlation analysis in combined SBMA groups $A$ and $B$ between alanine aminotransferase (ALT) and creatine kinase ( $r=0.63, p<0.0001$ ) (C), ALT and liver dome IHTG ( $r=0.31, p=0.09$ ) (D), liver dome IHTG and body mass index (BMI) $(r=0.44, p=0.01)(E)$, and liver dome IHTG and CAG repeat length $(r=0.32, p=0.08)(F)$.

correlated significantly with liver fat (IHTG) in participants with SBMA $(r=0.43, p=0.01$; figure $1 \mathrm{~B})$. The average thigh muscle triacylglycerol content was significantly higher in the SBMA group (30 $\pm 24 \%$ ) compared to the controls $(6 \pm 4 \%, p<0.001)$ and female carriers $(6 \pm 3 \%, p=0.002)$. 
Liver sample analysis. Four participants with SBMA consented to undergo liver biopsies (table e-1 at Neurology. org). Two had steatosis with minimal inflammation and no fibrosis (figure 2A). The 2 other participants had steatohepatitis, one with mild ballooning injury but without fibrosis (figure 2, B and C) and one with prominent ballooning hepatocellular injury, MalloryDenk bodies, and perisinusoidal fibrosis (figure 2, D-F).

Liver biopsy samples were further analyzed from 3 healthy controls, participants with NASH, and participants with SBMA. Hepatic expression of the AR by ddPCR was reduced by $85 \%$ in the SBMA samples compared to normal liver control samples $(p<0.05)$, whereas the non-SBMA NASH samples showed an intermediate reduction of $55 \%(\mathrm{n}=3$ for each group; figure $3 \mathrm{~A}$ ). Immunohistochemical staining for the AR showed nuclear localization within the hepatocytes (figure 3B). AR staining in the patient tissues showed patchy areas of increased nuclear staining compared to control samples. We observed most of the AR staining in the nonsteatotic periportal hepatocytes (zone 1).

To better describe the molecular pathways that are affected, we analyzed hepatic gene expression using

Figure 2 Histologic findings in spinal and bulbar muscular atrophy liver samples
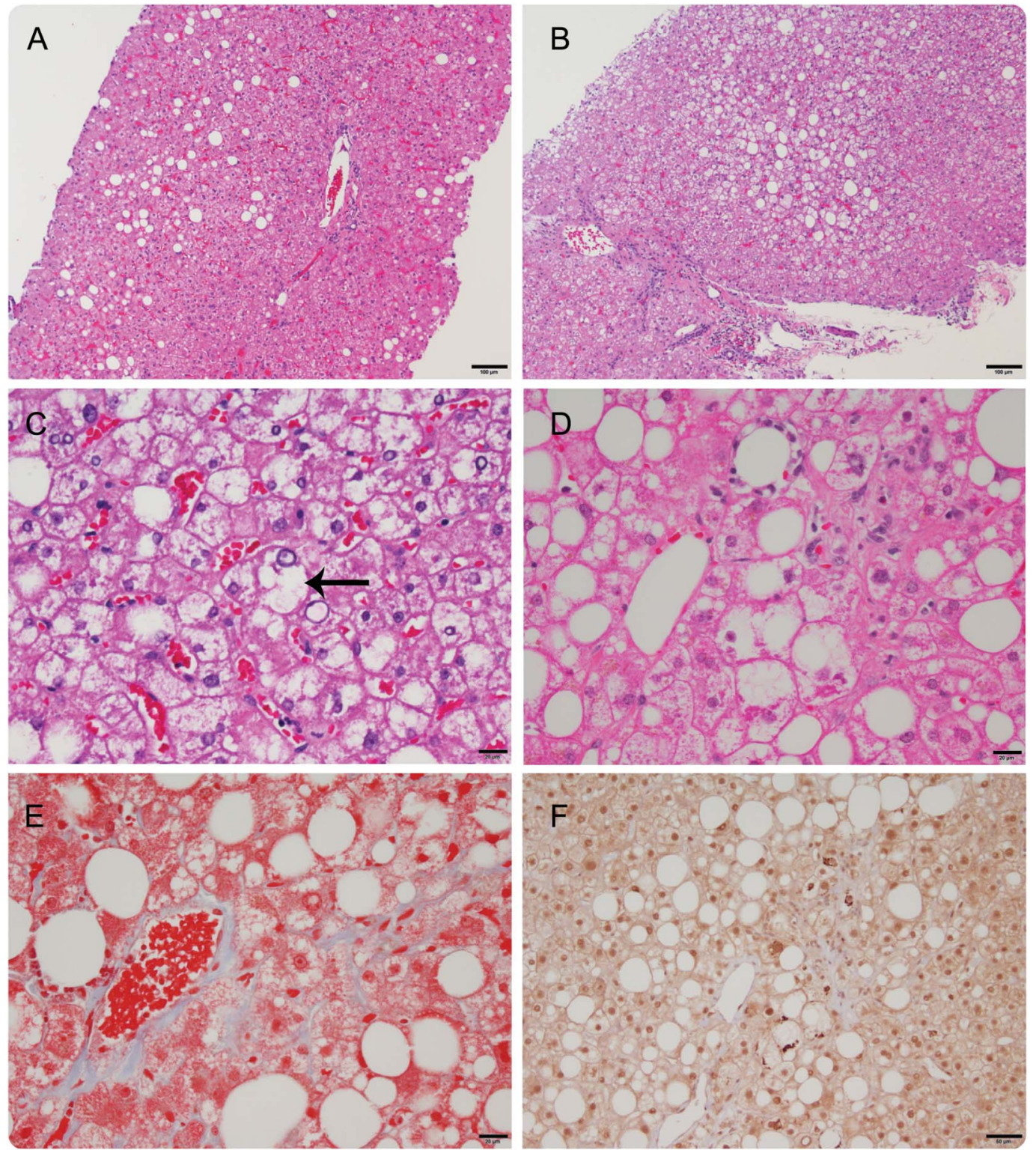

Participant SB4 liver sample hematoxylin \& eosin (H\&E) image shows mild steatosis with no ballooning and minimal inflammation (A). Participant SB6 H\&E staining shows mild steatosis, hepatocyte ballooning (arrow), and mild portal inflammation (B, C). Participant SB10 H\&E shows mild to moderate steatosis in zone 3 with hepatocyte ballooning, Mallory-Denk bodies, and inflammation (D-F). Mild perisinusoidal fibrosis is seen on Masson trichrome (E). Ubiquitin immunostaining shows staining of Mallory-Denk bodies, which appear as darkly stained irregular inclusions (F). Scale bar for $A$ and $B=100 \mu m$; $C, D$, and $E=20 \mu \mathrm{m} ; \mathrm{F}=50 \mu \mathrm{m}$. 


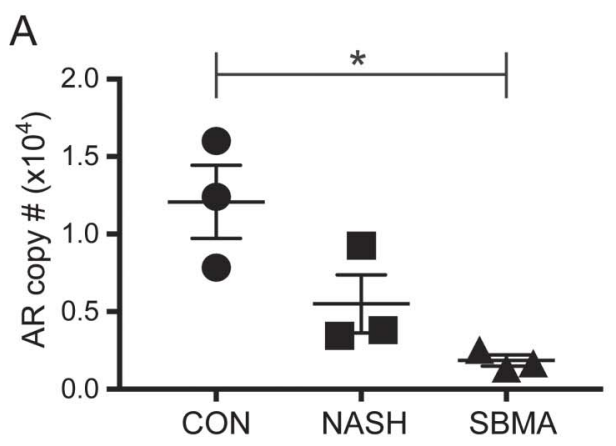

B

SBMA
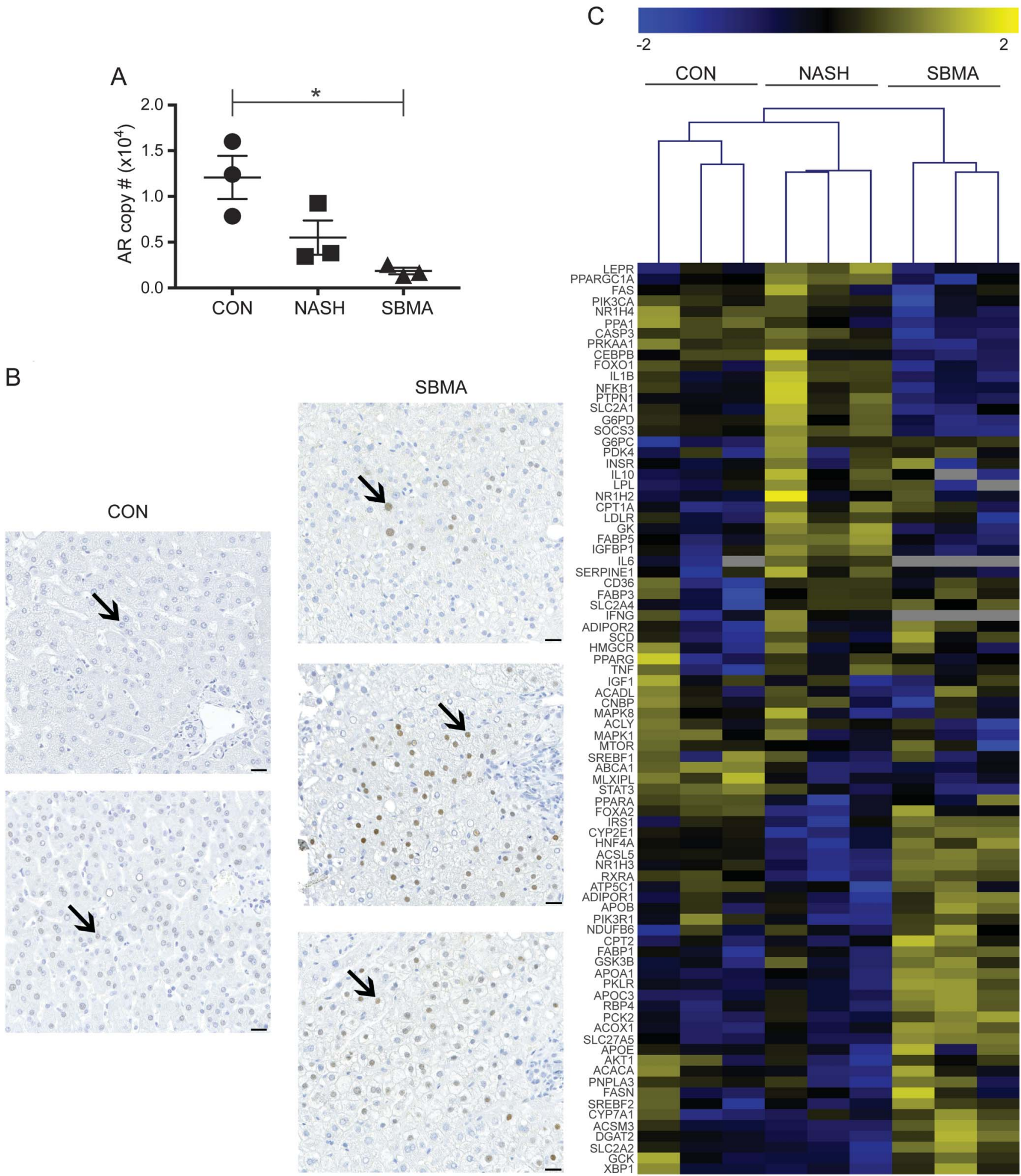

Digital droplet PCR shows a reduction in androgen receptor (AR) mRNA levels in SBMA liver samples compared to healthy and nonalcoholic steatohepatitis (NASH) control males (A); $* p<0.05$. Androgen receptor antibody immunohistochemistry staining in SBMA liver samples shows nuclear localization (arrows) and increased staining in SBMA compared to healthy control males (scale bar $=25 \mu \mathrm{m}$ ) (B). Hierarchical analysis with heat map shows gene expression patterns for the fatty liver disease signaling pathways in the 3 control, NASH, and SBMA liver samples (C).

a targeted array of 84 genes that had been previously characterized in fatty liver disease. Overall, $72 \%$ of the genes present in the array contain an androgen responsive element. ${ }^{20}$ The gene expression pattern in SBMA was different from both normal liver controls and from those with non-SBMA NASH 
(figure 3C). Differentially expressed genes included those involved in $\beta$-oxidation, insulin signaling, and adipokine signaling (figure e-1). Retinol binding protein 4 (RBP4) expression levels were increased in the SBMA samples compared to controls. The SBMA samples had less activation of inflammatory response and apoptosis pathways compared to NASH.

DISCUSSION We report near-universal excess deposition of triacylglycerol in the liver as part of the disease spectrum in SBMA, which is typically considered a neuromuscular disease. Participants with SBMA had additional features of the metabolic syndrome beyond fatty liver, including elevated glucose, insulin, and serum triglyceride. Studies of larger groups of patients with SBMA have also identified elevations in serum cholesterol levels, ${ }^{9}$ but our population size of 14 may have limited our ability to detect it. Interestingly, features of metabolic syndrome and liver fat content were present in patients with SBMA despite BMI values that were similar to controls. For the carriers, normal MRS hepatic triacylglycerol and laboratory findings suggest that liver function is not affected. The control group was enriched for African Americans, which may have contributed to the lower rate of NAFLD, as previously reported. ${ }^{21}$ However, the extremely high prevalence of fatty liver in the participants with SBMA is higher than any reported population-based estimates, including another analysis of healthy volunteers at the NIH Clinical Center, where $28 \%$ had NAFLD. ${ }^{22}$

Several features of the fatty liver in the SBMA suggest a direct association with the underlying disease: the nearuniversal presence of excess fat deposition, the presence of fatty liver despite normal BMI, and the difference in hepatic gene expression pathways involved in fatty liver disease between patients with SBMA and those with typical NASH (figure e-1).

The association of liver and thigh muscle fat in the SBMA cohort suggests that these 2 tissues may be similarly affected in the disease. Primary muscle ${ }^{23,24}$ and motor neuron degeneration ${ }^{25}$ have both been implicated in the pathogenesis of the disease, and toxicity may occur in the liver at a similar rate. However, whether SBMA causes fatty liver through a direct effect on the liver or indirectly from other organs is unknown. Impairment in insulin sensitivity and alterations in skeletal muscle insulin receptor levels have previously been reported in SBMA. ${ }^{9}$ Furthermore, decreased levels of activity due to the neuromuscular degeneration could also contribute to skeletal muscle insulin resistance; this in turn may contribute to increases in blood glucose and drive adipose tissue and hepatic lipogenesis. The fatty liver change may be a direct consequence of insulin resistance in the liver or skeletal muscle, and future studies will be needed to look for similar findings in other neuromuscular diseases with impaired fasting glucose. Interestingly, we did not detect a relationship in the SBMA cohort between muscle triacylglycerol content and HOMAIR. Insulin-like growth factor 1 (IGF-1) has important trophic effects on the muscle and spinal cord ${ }^{26,27}$ and is mostly synthesized in the liver. We have found low serum IGF-1 levels in a larger study of 56 patients with SBMA and 170 controls (unpublished), and it is possible that the IGF-1 reduction is a cause or effect of NAFLD. ${ }^{28}$

The alteration in AR function may predispose to fatty liver disease. Our patient samples have reduced AR transcript levels; however, immunohistochemical staining showed a patchy increase in the amount of AR protein. This suggests that a loss of function, toxic gain of function, or combination of both may result in the fatty liver finding. Mice with conditional knockout of hepatic AR have been reported to develop liver steatosis and insulin resistance, indicating that loss of normal AR function could be contributing in patients. ${ }^{29}$ Furthermore, these mice showed alterations in metabolism of the skeletal muscle, white adipose tissue, and liver, expanding the role of the liver AR function in metabolic homeostasis in multiple organ systems. Similarly, studies of serum androgen levels in men have shown impairments in testosterone levels associated with NAFLD. ${ }^{30,31}$ Our finding of increased RBP4 expression is consistent with a previous observation in adipocytes, where RBP4 expression was significantly increased with AR knockout. ${ }^{32}$ The RBP4 gene contains an androgen response element, ${ }^{20}$ providing a potential substrate for the mutant $A R$ to cause altered RBP4 expression. Impairments in autophagy and endoplasmic reticulum stress may also be responsible for increased triacylglycerol storage in SBMA liver. ${ }^{33-37}$ An association between higher free testosterone and NAFLD in a large cohort of premenopausal women also suggests a potential role for selective AR gain of function in mediating fatty liver changes. ${ }^{38}$ However, this relationship appears to be reversed in men, in whom lower levels of testosterone ${ }^{39}$ and sex hormone binding globulin ${ }^{40}$ were found to be associated with fatty liver disease.

This study has important implications for the management of patients with SBMA. The liver serves a crucial role in drug metabolism, and those prescribing medications or evaluating candidate therapeutics for patients with SBMA should be aware of the risk for fatty liver disease and monitor hepatic function. Furthermore, patients with SBMA often present with elevated liver enzymes. Importantly, we did not detect any patient with SBMA with features of end stage liver disease, and although most participants were found to have steatosis, only one had an NFS score suggestive of fibrosis and more advanced 
disease. Whether the consequences of fatty liver changes in SBMA are milder than typical NAFLD remains to be seen. The diagnosis of NASH requires histologic evaluation, and the small number of liver biopsies performed in this study limits our understanding of how frequently NASH occurs in this population. Noninvasive assessments of severity of NAFLD/NASH are limited in their accuracy; however, although most participants were found to have steatosis, only one had an NFS score suggestive of fibrosis and advanced disease. The liver is accessible to frequent monitoring with MRS and ultrasound testing, which may serve as a biomarker to monitor in future studies. Furthermore, the study of mutant $\mathrm{AR}$ in liver tissue of patients with SBMA and animal models may be a useful tool for further investigating the disease mechanism of SBMA and NAFLD.

\section{AUTHOR CONTRIBUTIONS}

R.D. Guber and Dr. Takyar: acquisition of data, analysis of data, and drafting of the manuscript. A. Kokkinis and Dr. Fox: study design, acquisition of data, and revising of the manuscript. Drs. Alao, Remaley, Hewitt, Kleiner, Liu, and Hadigan: acquisition of data and revising of the manuscript. I. Kats and D. Bakar: analysis of data and revising of the manuscript. Dr. Fischbeck: revising of the manuscript and study design. Drs. Rotman and Grunseich: study design, acquisition of data, analysis of data, and drafting of the manuscript.

\section{ACKNOWLEDGMENT}

The authors thank Elizabeth Hartnett, MSW (NINDS, NIH), for her help in scheduling participants during the study; the clinical staff of the outpatient NIH neurology and gastroenterology clinics for their assistance during the participants' visits; the research participants; and the Liver Tissue Cell Distribution System at the University of Minnesota for providing control and NASH liver samples. The authors thank Dr. Nicholas Maragakis (Johns Hopkins University) for his help as a safety monitor during this study.

\section{STUDY FUNDING}

Study funded by intramural research funds from the National Institute of Neurologic Disorders and Stroke, National Institute of Diabetes and Digestive and Kidney Diseases, National Institute of Allery and Infectious Diseases, and National Cancer Institute.

\section{DISCLOSURE}

The authors report no disclosures relevant to the manuscript. Go to Neurology.org for full disclosures.

Received June 6, 2017. Accepted in final form September 19, 2017.

\section{REFERENCES}

1. Kennedy WR, Alter M, Sung JH. Progressive proximal spinal and bulbar muscular atrophy of late onset: a sexlinked recessive trait. Neurology 1968;18:671-680.

2. La Spada AR, Wilson EM, Lubahn DB, Harding AE, Fischbeck KH. Androgen receptor gene mutations in Xlinked spinal and bulbar muscular atrophy. Nature 1991; 352:77-79.

3. Hashizume A, Katsuno M, Banno H, et al. Longitudinal changes of outcome measures in spinal and bulbar muscular atrophy. Brain 2012;135:2838-2348.

4. Rhodes LE, Freeman BK, Auh S, et al. Clinical features of spinal and bulbar muscular atrophy. Brain 2009;132: 3242-3251.
5. Ishihara H, Kanda F, Nishi H, Sumino K, Chihara K. Clinical features and skewed X-chromosome inactivation in female carriers of $\mathrm{X}$-linked recessive spinal and bulbar muscular atrophy. J Neurol 2001;248: 856-860.

6. Chevalier-Larsen ES, O'Brien CJ, Wang H, et al. Castration restores function and neurofilament alterations of aged symptomatic males in a transgenic mouse model of spinal and bulbar muscular atrophy. J Neurosci 2004;24: 4778-4786.

7. Querin G, Bertolin C, Da Re E, et al. Non-neural phenotype of spinal and bulbar muscular atrophy: results from a large cohort of Italian patients. J Neurol Neurosurg Psychiatry 2016;87:810-816.

8. Araki A, Katsuno M, Suzuki K, et al. Brugada syndrome in spinal and bulbar muscular atrophy. Neurology 2014;82: 1813-1821.

9. Nakatsuji H, Araki A, Hashizume A, et al. Correlation of insulin resistance and motor function in spinal and bulbar muscular atrophy. J Neurol 2017;264:839-847.

10. Vernon G, Baranova A, Younossi ZM. Systematic review: the epidemiology and natural history of non-alcoholic fatty liver disease and non-alcoholic steatohepatitis in adults. Aliment Pharmacol Ther 2011;34:274-285.

11. Angulo P, Kleiner DE, Dam-Larsen S, et al. Liver fibrosis, but no other histologic features, is associated with longterm outcomes of patients with nonalcoholic fatty liver disease. Gastroenterology 2015;149:389-397.

12. Chalasani N, Younossi Z, Lavine JE, et al. The diagnosis and management of non-alcoholic fatty liver disease: practice guideline by the American Association for the Study of Liver Diseases, American College of Gastroenterology, and the American Gastroenterology Association. Hepatology 2012;55:2005-2023.

13. American Association for the Study of Liver Diseases Practice Guidelines. Available at: aasld.org. Accessed July 2017.

14. European Association for the Study of the Liver (EASL), European Association for the Study of Diabetes (EASD), European Association for the Study of Obesity (EASO). EASL-EASD-EASO Clinical Practice Guidelines for the management of non-alcoholic fatty liver disease. J Hepatol 2016;64:1388-1402.

15. 2015-2020 Dietary Guidelines for Americans. Rockville, MD: US Department of Health and Human Services and US Department of Agriculture; 2015.

16. Georgoff P, Thomasson D, Louie A, et al. Hydrogen-1 MR spectroscopy for measurement and diagnosis of hepatic steatosis. Am J Roentgenol 2012;199:2-7.

17. Longo R, Pollesello P, Ricci C, et al. Proton MR spectroscopy in quantitative in vivo determination of fat content in human liver steatosis. J Magn Reson Imaging 1995;5:281-285.

18. Kleiner DE, Brunt EM, Van Natta M, et al. Design and validation of a histological scoring system for nonalcoholic fatty liver disease. Hepatology 2005;41:1313-1321.

19. Angulo P, Hui JM, Marchesini G, et al. The NAFLD fibrosis score: a noninvasive system that identifies liver fibrosis in patients with NAFLD. Hepatology 2007;45: 846-854.

20. Wilson S, Qi J, Filipp FV. Refinement of the androgen response element based on ChIP-Seq in androgeninsensitive and androgen-responsive prostate cancer cell lines. Sci Rep 2016;6:32611.

21. Browning JD, Szczepaniak LS, Dobbins R, et al. Prevalence of hepatic steatosis in an urban population in the 
Unites States: impact of ethnicity. Hepatology 2004;40: 1387-1395.

22. Takyar V, Nath A, Beri A, Gharib AM, Rotman Y. How healthy are the "healthy volunteers"? Penetrance of NAFLD in the biomedical research volunteer pool. Hepatology 2017;66:825-833.

23. Lieberman AP, Yu Z, Murray S, et al. Peripheral androgen receptor gene suppression rescues disease in mouse models of spinal and bulbar muscular atrophy. Cell Rep 2014;7: 774-784.

24. Cortes CJ, Ling SC, Guo LT, et al. Muscle expression of mutant androgen receptor accounts for systemic and motor neuron disease phenotypes in spinal and bulbar muscular atrophy. Neuron 2014;82: 295-307.

25. Abel A, Walcott J, Woods J, Duda J, Merry DE. Expression of expanded repeat androgen receptor produces neurologic disease in transgenic mice. Hum Mol Genet 2001; 10:107-116.

26. Rinaldi C, Bott LC, Chen KL, et al. Insulin like growth factor (IGF)-1 administration ameliorates disease manifestations in a mouse model of spinal and bulbar muscular atrophy. Mol Med 2012;18:1261-1268.

27. Palazzolo I, Stack C, Kong L, et al. Overexpression of IGF-1 in muscle attenuates disease in a mouse model of spinal and bulbar muscular atrophy. Neuron 2009;63: 316-328.

28. Arturi F, Succurro E, Procopio C, et al. Nonalcoholic fatty liver disease is associated with low circulating levels of insulin-like growth factor-1. J Clin Endocrinol Metab 2011;96:E1640-E1644.

29. Lin HY, Yu IC, Wang RS, et al. Increased hepatic steatosis and insulin resistance in mice lacking hepatic androgen receptor. Hepatology 2008;47:1924-1935.

30. Kim S, Kwon H, Park JH, et al. A low level of serum total testosterone is independently associated with nonalcoholic fatty liver disease. BMC Gastroenterol 2012;12:69.
31. Volzke H, Aumann N, Krebs A, et al. Hepatic steatosis is associated with low serum testosterone and high serum DHEAS levels in men. Int J Androl 2010;33:45-53.

32. McInnes KJ, Smith LB, Hunger NI, Saunders P. Deletion of the androgen receptor in adipose tissue in male mice elevates retinol binding protein 4 and reveals independent effects on visceral fat mass and on glucose homeostasis. Diabetes 2012;61:1072-1081.

33. Chua JP, Reddy SL, Merry DE, et al. Transcriptional activation of TFEB/ZKSCAN3 target genes underlies enhanced autophagy in spinobulbar muscular atrophy. Hum Mol Genet 2014;23:1376-1386.

34. Singh R, Kaushik S, Wang Y, et al. Autophagy regulates lipid metabolism. Nature 2009;458:1131-1137.

35. Bozaykut P, Sahin A, Karademir B, Ozer NK. Endoplasmic reticulum stress related molecular mechanisms in nonalcoholic steatohepatitis. Mech Ageing Dev 2016;157:17-29.

36. Rutkowski DT, Wu J, Back SH, et al. UPR pathways combine to prevent hepatic steatosis caused by ER stressmediated suppression of transcriptional master regulators. Dev Cell 2008;15:829-840.

37. Yu Z, Wang AM, Adachi, et al. Macroautophagy is regulated by the UPR-mediator CHOP and accentuates the phenotype of SBMA mice. PLoS Genet 2011;7:e1002321.

38. Sarkar M, Weelons M, Cedars MI, et al. Testosterone levels in pre-menopausal women are associated with nonalcoholic fatty liver disease in midlife. Am J Gastroenterol 2017;112:755-762.

39. Barbonetti A, Vassallo MRC, Cotugno M, Felzani G, Francavilla S, Francavilla F. Low testosterone and nonalcoholic fatty liver disease: evidence for their independent association in men with chronic spinal cord injury. J Spinal Cord Med 2016;39:443-449.

40. Lazo M, Zeb I, Nasir K, et al. (MESA AC 291) the association of endogenous sex hormones with liver fatmulti-ethnic study of atherosclerosis (MESA). Clin Gastroenterol Hepatol 2015;13:1686-1693.

\section{Pit Neurology.org Offers Important Information to Patients and Their Families}

The Neurology ${ }^{\circledR}$ Patient Page provides:

- A critical review of ground-breaking discoveries in neurologic research that are written especially for patients and their families

- Up-to-date patient information about many neurologic diseases

- Links to additional information resources for neurologic patients

All Neurology Patient Page articles can be easily downloaded and printed, and may be reproduced to distribute for educational purposes. Click on the 'Patients' link on the home page (Neurology.org) for a complete index of Patient Pages. 


\title{
Neurology
}

\author{
Nonalcoholic fatty liver disease in spinal and bulbar muscular atrophy \\ Robert D. Guber, Varun Takyar, Angela Kokkinis, et al. \\ Neurology 2017;89;2481-2490 Published Online before print November 15, 2017 \\ DOI 10.1212/WNL.0000000000004748
}

This information is current as of November 15, 2017

Neurology ${ }^{\circledR}$ is the official journal of the American Academy of Neurology. Published continuously since 1951, it is now a weekly with 48 issues per year. Copyright Copyright (C) 2017 The Author(s). Published by Wolters Kluwer Health, Inc. on behalf of the American Academy of Neurology.. All rights reserved. Print ISSN: 0028-3878. Online ISSN: 1526-632X.

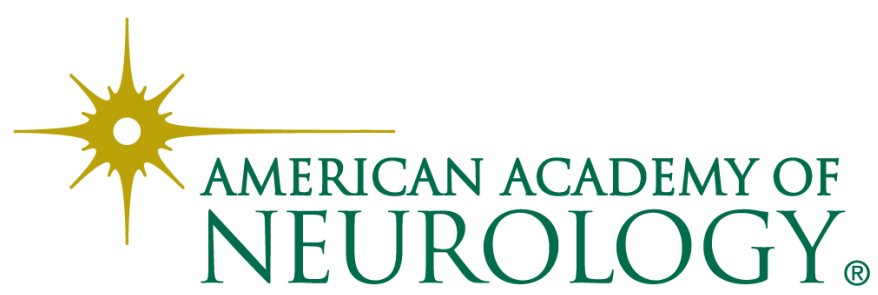




\section{Updated Information \& Services}

\section{Supplementary Material}

References

Citations

Subspecialty Collections

Permissions \& Licensing

\section{Reprints}

including high resolution figures, can be found at: http://n.neurology.org/content/89/24/2481.full

Supplementary material can be found at: http://n.neurology.org/content/suppl/2017/11/15/WNL.0000000000004 748.DC1

This article cites 38 articles, 5 of which you can access for free at: http://n.neurology.org/content/89/24/2481.full\#ref-list-1

This article has been cited by 1 HighWire-hosted articles: http://n.neurology.org/content/89/24/2481.full\#\#otherarticles

This article, along with others on similar topics, appears in the following collection(s):

\section{Anterior nerve cell disease}

http://n.neurology.org/cgi/collection/anterior_nerve_cell_disease

\section{Cohort studies}

http://n.neurology.org/cgi/collection/cohort_studies

\section{MRS}

http://n.neurology.org/cgi/collection/mrs

Muscle disease

http://n.neurology.org/cgi/collection/muscle_disease

Trinucleotide repeat diseases

http://n.neurology.org/cgi/collection/trinucleotide_repeat_diseases

Information about reproducing this article in parts (figures,tables) or in its entirety can be found online at:

http://www.neurology.org/about/about_the_journal\#permissions

Information about ordering reprints can be found online:

http://n.neurology.org/subscribers/advertise

Neurology ${ }^{\circledR}$ is the official journal of the American Academy of Neurology. Published continuously since 1951, it is now a weekly with 48 issues per year. Copyright Copyright ( 2017 The Author(s). Published by Wolters Kluwer Health, Inc. on behalf of the American Academy of Neurology.. All rights reserved. Print ISSN: 0028-3878. Online ISSN: 1526-632X.

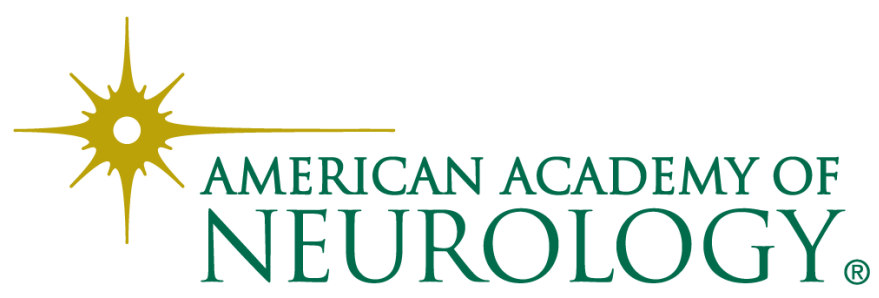

